\title{
Estudo do comportamento da PIO de 24 horas e frequência de glaucoma em pacientes com síndrome da apnéia obstrutiva do sono
}

\author{
Study on 24 hours IOP study and glaucoma frequency \\ in patients with obstructive sleep apnea syndrome
}

Maria Rosa Bet de Moraes Silva', Silke Anna Theresa Weber²

\section{ResUmo}

Objetivo: Determinar a frequência de glaucoma e avaliar o comportamento da pressão intraocular (PIO) de 24 horas em pacientes portadores da síndrome da apnéia obstrutiva do sono (SAOS). Métodos: Foi realizado estudo transversal em 11 pacientes consecutivos portadores de SAOS diagnosticados por polissonografia. Foram analisados dados demográficos: idade, sexo, raça/cor, peso, altura e doenças associadas. Os pacientes foram submetidos à avaliação oftalmológica completa que incluiu campo visual e também avaliação da pressão intraocular (PIO) de 24 horas com tonômetro de aplanação às 9h, 12h, 15h, 18h, 24h e 6h deitado e sentado. O critério para diagnóstico de glaucoma foi alteração de campo visual (CV) compatível com glaucoma e uma ou mais das seguintes alterações: relação escavação disco $\geq 0,7$, hemorragia, defeito em cunha, vaso em baioneta, sinal de Hoyt, assimetria > que 0,2 entre as escavações dos dois olhos. O ângulo devia ser aberto sem alterações. Resultados: $9(82 \%)$ de 11 pacientes apresentaram glaucoma ou suspeita de glaucoma, sendo $9 \%$ glaucoma de pressão normal e $73 \%$ suspeita de glaucoma por apresentarem alterações do nervo óptico ou hipertensão ocular. A média dos valores de PIO dos 11 pacientes apresentou-se mais elevada às 6 horas quando os pacientes estavam deitados. Ocorreram flutuações de $\mathrm{PIO} \geq 5$ mmHg em 7 (64\%) dos pacientes sendo que ocorreram flutuações de até $14 \mathrm{mmHg}$ e picos de PIO de até $32 \mathrm{mmHg}$. Conclusão: A SAOS pode ser um fator de risco importante para o esenvolvimento de glaucoma principalmente de pressão normal. Pacientes com SAOS devem ser referidos para o oftalmologista e os oftalmologistas devem estar atentos para a associação de distúrbios do sono em pacientes com glaucoma de ângulo aberto.

Descritores: Apnéia do sono tipo obstrutiva; Pressão intra-ocular; Glaucoma de ângulo aberto; Técnicas de diagnóstico oftalmológico

\footnotetext{
${ }^{1}$ Livre-docente, Professora Adjunta do Departamento de Oftalmologia, Otorrinolaringologia e Cirurgia de Cabeça e Pescoço da Faculdade de Medicina de Botucatu da Universidade Estadual "Julio de Mesquita Filho" - UNESP - Botucatu (SP), Brasil;

${ }^{2}$ Doutora, Professora do Departamento de Oftalmologia, Otorrinolaringologia e Cirurgia de Cabeça e Pescoço da Faculdade de Medicina de Botucatu da Universidade Estadual "Julio de Mesquita Filho" - UNESP - Botucatu (SP), Brasil.
}

Hospital das Clínicas da Faculdade de Medicina de Botucatu da Universidade Estadual "Julio de Mesquita Filho" - UNESP Botucatu (SP), Brasil

Recebido para publicação em: 22/4/2009 - Aceito para publicação em 13/11/2009 


\section{INTRODUÇÃO}

A prevalência do glaucoma varia conforme a população estudada, mas é consenso estar por volta de $2 \%$ na população branca acima de 40 $\operatorname{anos}^{(1)}$. A pressão intraocular é o principal fator de risco para o glaucoma, mas déficits microcirculatórios, imunidade alterada, excitotoxicidade dentre outros, podem também estar implicados isoladamente ou de forma combinada $^{(2,3)}$.

A literatura tem mostrado que a prevalência de glaucoma em portadores de apnéia obstrutiva do sono é maior do que a da população em geral ${ }^{(4-6)}$. Mojon et al. ${ }^{(4)}$ observaram $5(7,2 \%)$ pacientes com glaucoma em 69 portadores de síndrome de apnéia do sono. Dos cinco, três tinham glaucoma de pressão normal e 2 glaucoma primário de ângulo aberto. Outros estudos sugerem associação da apnéia do sono com glaucoma ${ }^{(7-9)}$. No entanto esta associação não tem sido confirmada por outros autores. ${ }^{(10,11)}$

Tendo em vista as contradições sobre a associação de glaucoma e síndrome da apnéia obstrutiva (SAOS) e a falta de estudos de avaliação de pressão intra-ocular (principal fator de risco do glaucoma) em portadores de SAOS, este estudo teve como objetivos: determinar a frequência de glaucoma e avaliar o comportamento da pressão intra-ocular (PIO) de 24 horas em pacientes portadores da síndrome da apnéia obstrutiva do sono.

\section{MÉTOdos}

Foram estudados 11 pacientes consecutivos, portadores da síndrome da apnéia obstrutiva do sono com diagnóstico polissonográfico do Ambulatório de Distúrbios do Sono do HC da Faculdade de Medicina de Botucatu - UNESP atendidos no período de abril à dezembro de 2006.

Foram analisados os dados demográficos dos pacientes: idade, sexo, raça/cor, peso, altura, índice de massa corporal e doenças associadas.

Todos os pacientes realizaram polissonografia de noite inteira. A SAOS foi diagnosticada quando o índice de distúrbios respiratórios (RDI) era maior do que 5. Do laudo da polissonografia foram analisados os dados de RDI, eficiência do sono, saturação média de $\mathrm{O}_{2}$ e índice de despertar para efeito de diagnóstico de SAOS ${ }^{(12,13)}$.

Todos os pacientes foram submetidos a exame oftalmológico completo que incluiu: história e antecedentes, medida da acuidade visual (AV) a 6 metros com tabela de Snellen, refração dinâmica, biomicroscopia do segmento anterior, biomicroscopia do segmento posterior com lente de Volk de 78D, campo visual (C.V.) com programa 24-2 do perímetro de Humphrey e gonioscopia. Foi realizada CTD (curva tensional diária - com 8 medidas de PIO: 6h deitado, 6h sentado 9h, 12h, $15 \mathrm{~h}, 18 \mathrm{~h}, 21 \mathrm{~h}$ e $24 \mathrm{~h}$ ). As medidas foram feitas com tonômetro de Goldman exceto a das 6h (deitado) que foi realizada com tonômetro de Perkins. Todas as avaliações da PIO foram feitas em ambiente hospitalar por um dos autores (MRBMS).

O critério utilizado para diagnóstico de glaucoma foi: alteração de C.V. de pelo menos 2 pontos adjacentes não periféricos com $\mathrm{p}<5 \%$ no gráfico "pattern deviation" associado a um ponto com $\mathrm{p}<1 \%$, em localização compatível com defeito glaucomatoso e GHT outside normal limits associada a uma ou mais das alterações do nervo óptico e camada de fibras nervosas: relação escavação disco $\geq 0,7$, hemorragia no disco, defeito em cunha, vaso em baioneta, sinal de Hoyt, assimetria de escavação $>0,2$, na presença de ângulo aberto sem alterações à gonioscopia.

O diagnóstico de glaucoma de pressão normal foi dado quando o paciente apresentava as alterações descritas acima associadas a $\mathrm{PIO}<21 \mathrm{mmHg}$.

O critério utilizado para suspeito de glaucoma foi: $\mathrm{CV}$ anormal, porém não confirmado com outro exame, $\mathrm{PIO}>21 \mathrm{mmHg}$, sem alteração de $\mathrm{CV}$ e de disco óptico, relação escavação / disco $\leq 0,6$, perda de padrão isn't, assimetrias de escavação $\leq 0,2$ com CV normal e ângulo aberto sem alterações à gonioscopia.

Os dados de PIO foram descritos como média (M) e desvio padrão (DP) e foram comparados os valores de média de PIO dos olhos direitos (OD) e olhos esquerdos (OE) por meio do teste t-Student de amostras pareadas, considerado significativo quando $\mathrm{p}<0,05$.

$\mathrm{O}$ estudo foi aprovado pelo Comitê de Ética em Pesquisa (CEP 38/2006) e todos os pacientes assinaram Termo de Consentimento Livre e Esclarecido.

\section{Resultados}

Dos 11 pacientes, 3 (27,2\%) eram do sexo feminino e $8(72,7 \%)$ do sexo masculino. A idade média dos 11 pacientes foi de 51,2 anos, sendo a das mulheres de 51,3 anos e a dos homens de 51,2. Dos 11 pacientes, 9 eram brancos e 2 negros/pardos (Tabela 1).

Quanto ao RDI, 5 pacientes apresentaram RDI > $30 \mathrm{n} / \mathrm{h}$ (número de distúrbios respiratórios por hora), considerado SAOS grave, 5 pacientes RDI entre 15 e $30 \mathrm{n} /$ $\mathrm{h}$, considerado SAOS moderada e somente 1 paciente apresentou SAOS leve com RDI entre 5 a $15 \mathrm{n} / \mathrm{h}$.

$\mathrm{O}$ valor da média do índice de massa corporal (IMC) foi de $33,56 \mathrm{Kg} / \mathrm{m}^{2}$. Considerando-se como obesidade IMC $>30 \mathrm{Kg} / \mathrm{m}^{2}$, todos os pacientes individualmente apresentaram IMC compatível com obesidade, exceto o de número $5\left(\mathrm{IMC}=24,98 \mathrm{Kg} / \mathrm{m}^{2}\right)$. 
Tabela 1

Caracterização dos 11 portadores de SAOS (síndrome da apnéia obstrutiva do sono) quanto à idade (anos) sexo, raça, IMC e doenças sistêmicas associadas (UNESP - Botucatu - 2006)

\begin{tabular}{cccccc}
\hline Paciente & Idade (anos) & Sexo & Raça & IMC & Doenças sistêmicas \\
\hline 1 & 41 & M & B & 39,87 & - \\
2 & 52 & M & B & 38,69 & Doença coronária \\
3 & 50 & F & N & 36,41 & HA \\
4 & 67 & M & B & 30,86 & HA \\
5 & 63 & M & B & 24,98 & HA, artrite reumatióide \\
6 & 57 & M & P & 33,59 & Diabetes \\
7 & 48 & F & B & 31,24 & Doença coronária \\
8 & 35 & M & B & 33,83 & - \\
9 & 56 & F & B & 25,089 & HA \\
10 & 48 & M & B & 33,31 & Hanseníase \\
11 & 47 & M & B & 37,27 & HA \\
\hline MÉDIA & 51,27 & & & 33,56 & \\
\hline
\end{tabular}

M- masculino; F-feminino; B- branco; P- pardo; $\mathrm{N}$ - negro; IMC- indice de massa corporal $\left(\mathrm{Kg} / \mathrm{m}^{2}\right)$; HA - hipertensão arterial

Tabela 2

Valores de pressão intraocular (PIO) em mmHg de ambos os olhos nos diferentes horários, média e desvio-padrão da média da $\mathrm{PIO}(\mathrm{mmHg})$ de ambos os olhos e valores de $p$ em portadores de SAOS (UNESP - Botucatu - 2006)

\begin{tabular}{|c|c|c|c|c|c|c|c|c|c|c|c|c|c|c|c|c|}
\hline & \multicolumn{2}{|c|}{$9 h$} & \multicolumn{2}{|c|}{$12 \mathrm{~h}$} & \multicolumn{2}{|c|}{$15 \mathrm{~h}$} & \multicolumn{2}{|c|}{$18 \mathrm{~h}$} & \multicolumn{2}{|c|}{$21 \mathrm{~h}$} & \multicolumn{2}{|c|}{$24 h$} & \multicolumn{2}{|c|}{ 6h D } & \multicolumn{2}{|c|}{ 6h S } \\
\hline & OD & $\mathbf{O E}$ & OD & $\mathbf{O E}$ & OD & OE & OD & $\mathbf{O E}$ & OD & $\mathbf{O E}$ & OD & OE & OD & OE & OD & OE \\
\hline 1 & 17 & 16 & 20 & 22 & 17 & 18 & 19 & 18 & 15 & 11 & 19 & 14 & 21 & 16 & 25 & 23 \\
\hline 2 & 19 & 18 & 20 & 21 & 20 & 22 & 20 & 19 & 18 & 23 & 17 & 17 & 20 & 20 & 18 & 17 \\
\hline 3 & 15 & 14 & 16 & 16 & 14 & 14 & 14 & 15 & 12 & 12 & 12 & 15 & 14 & 13 & 14 & 13 \\
\hline 4 & 16 & 14 & 13 & 15 & 16 & 15 & 15 & 14 & 15 & 14 & 16 & 12 & 15 & 17 & 14 & 16 \\
\hline 5 & 14 & 14 & 14 & 14 & 14 & 14 & 13 & 13 & 15 & 14 & 16 & 14 & 16 & 17 & 16 & 14 \\
\hline 6 & 17 & 17 & 12 & 13 & 11 & 12 & 13 & 12 & 11 & 12 & 10 & 10 & 20 & 20 & 15 & 15 \\
\hline 7 & 20 & 21 & 15 & 15 & 14 & 14 & 14 & 13 & 18 & 21 & 19 & 24 & 28 & 28 & 20 & 21 \\
\hline 8 & 24 & 24 & 20 & 18 & 20 & 20 & 20 & 20 & 21 & 21 & 19 & 19 & 24 & 32 & 24 & 27 \\
\hline 9 & 9 & 8 & 12 & 12 & 14 & 12 & 14 & 12 & 11 & 10 & 12 & 12 & 13 & 12 & 14 & 12 \\
\hline 10 & 10 & 10 & 14 & 14 & 17 & 15 & 16 & 14 & 15 & 13 & 19 & 18 & 25 & 25 & 19 & 20 \\
\hline 11 & 12 & 12 & 12 & 12 & 10 & 10 & 9 & 9 & 8 & 8 & 8 & 14 & 8 & 10 & 14 & 12 \\
\hline $\mathrm{M}$ & 15,73 & 15,27 & 15,27 & 15,64 & 15,18 & 15,09 & 15,18 & 14,45 & 14,45 & 14,45 & 15,18 & 15,36 & 18,55 & 19,09 & 17,55 & 17,27 \\
\hline DP & 4,43 & 4,65 & 3,29 & 3,38 & 3,22 & 3,59 & 3,37 & 3,33 & 3,75 & 4,97 & 4,02 & 3,93 & 5,94 & 6,86 & 4,06 & 4,90 \\
\hline $\mathrm{p}$ & \multicolumn{2}{|c|}{0,095} & \multicolumn{2}{|c|}{0,306} & \multicolumn{2}{|c|}{0,809} & \multicolumn{2}{|c|}{0,023} & \multicolumn{2}{|c|}{1,000} & \multicolumn{2}{|c|}{0,826} & \multicolumn{2}{|c|}{0,573} & \multicolumn{2}{|c|}{0,625} \\
\hline
\end{tabular}

OD- olho direito; OE- olho esquerdo; M- média; DP- desvio padrão; p- comparação entre médias de OD e OE por teste de t de Student para amostras pareadas

Quanto às doenças associadas, foram encontrados: hipertensão arterial em 6, doença coronariana em 2 , diabetes em 1 , artrite reumatóide em 1 e hanseníase em 1. Apenas 2 pacientes não apresentavam nenhuma outra patologia (Tabela 1).

Todos os pacientes negavam ter glaucoma e negavam antecedentes de glaucoma na família.

A Tabela 2 apresenta os valores das medidas da PIO de 24 horas (9h, 12h, 15h, 18h, 21h, 24h, 6h deitado e $6 \mathrm{~h}$ sentado) de ambos os olhos ( $\mathrm{AO})$ dos 11 pacientes, assim como a média e desvio-padrão dos valores em cada um dos horários e valores de p da comparação dos valores de média de PIO entre os dois olhos. A média dos valores de PIO de 9 horas foi semelhante em AO, sendo de $15,73 \mathrm{mmHg}$ e $15,27 \mathrm{mmHg}$ respectivamente em OD e OE $(p=0,095)$. A análise estatística mostrou que não houve diferença entre a PIO de OD e OE, exceto às $18: 00 \mathrm{~h}(\mathrm{p}=0,02)$. A partir das $12 \mathrm{~h}$ observa-se que a PIO sofre ligeira queda que persiste até as $21 \mathrm{~h}$, quando se inicia elevação culminando com o pico às $6 \mathrm{~h}$ 
Tabela 3

Presença de glaucoma ou suspeita de glaucoma nos
portadores de Síndrome da apnéia obstrutiva do sono
- SAOS (UNESP - Botucatu - 2006).

\begin{tabular}{ccc}
\hline Paciente & \multicolumn{2}{c}{ Glaucoma/suspeito } \\
& OD & OE \\
\hline 1 & suspeito* $^{*}$ & suspeito* \\
2 & - & suspeito* \\
3 & - & suspeito \\
4 & suspeito & suspeito \\
5 & - & $\%$ \\
6 & GPN & suspeito \\
7 & suspeito* & suspeito* \\
8 & suspeito* & suspeito* \\
9 & suspeito & suspeito \\
10 & suspeito* & suspeito* \\
11 & - & $\%$
\end{tabular}

suspeito* - por apresentar pressão intraocular $>21 \mathrm{mmHg}$; suspeito - por apresentar alteracões de nervo óptico e campo visual;OD - olho direito; OE - olho esquerdo; GP̉ - glaucoma de pressão normal

(paciente deitado) cujos valores foram: $18,55 \mathrm{mmHg}$ para o OD e de 19,09 $\mathrm{mmHg}$ para o OE.

A média dos valores de PIO às $6 \mathrm{~h}$ com o paciente sentado foram menores do que os valores com o paciente deitado, sendo a diferença de $1 \mathrm{mmHg}$ para o OD e $1,82 \mathrm{mmHg}$ para o OE (Tabela 2 e Figura 1 ).

Dos 22 olhos dos 11 pacientes, 1 olho apresentou glaucoma de pressão normal (OD do $\left.n^{\circ} 6\right)$. Outros 6 olhos $\left(\mathrm{OE}-\mathrm{n}^{\circ} 3, \mathrm{AO}-\mathrm{n}^{\circ} 4, \mathrm{OE}-\mathrm{n}^{\circ} 6\right.$ e $\left.\mathrm{AO} \mathrm{n}^{\circ} 9\right)$ foram considerados suspeitos de glaucoma por apresentarem alterações do nervo óptico e de campo visual. Pacientes que apresentaram alterações apenas de CV não foram considerados suspeitos pelo fato do exame não ter sido repetido (Tabela 3).

Os pacientes de números 1, 2, 7, 8 e 10 apresentaram valores de $\mathrm{PIO}$ maiores do que $21 \mathrm{mmHg}$, mas não apresentaram alterações de nervo óptico e campo visual (Tabelas 2 e 3 ).

\section{Dıscussão}

A SAOS é uma doença comum, definida como obstrução intermitente das vias aéreas superiores durante o sono, associada à hipóxia, variação de pressão intratorácica, ativação simpática e fragmentação do sono. Esses fenômenos são responsáveis por repercussões cardiovasculares, que incluem hipertensão arterial e pulmonar, arritmias cardíacas, infarto do miocárdio e neurológicas como o acidente vascular cerebral ${ }^{(13-15)}$.

A SAOS também tem sido apontada como fator de risco para o desenvolvimento do glaucoma principalmente do glaucoma de pressão normal ${ }^{(6,7)}$.

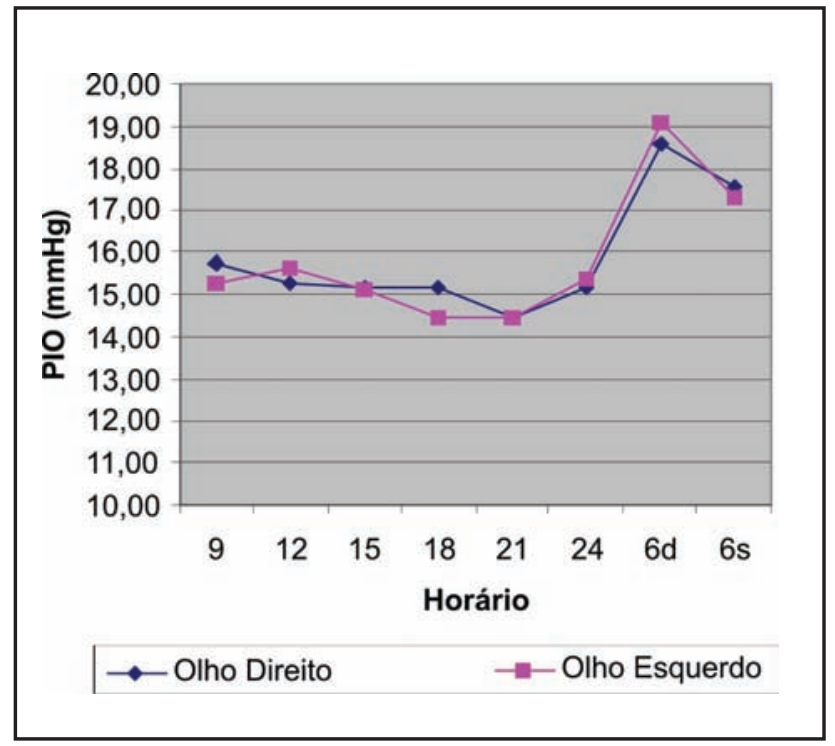

Figura 1: Curva tensional diária 8 medidas de pressão intraocular (PIO) em mmHg de ambos os olhos de portadores da Síndrome da apnéia obstrutiva do sono - SAOS (UNESP - Botucatu - 2006)

No presente estudo que inclui pacientes brancos e negros foi encontrada frequência de glaucoma de $4,5 \%$ ( 1 de 22 olhos) e considerando-se frequência por paciente foi de $9 \%$ ( 1 de 11 pacientes).

A prevalência de glaucoma em portadores de SAOS varia de 4,4 a 7,2\% na população branca ${ }^{(4-6)}$ mas não há nenhum estudo que inclua negros na amostra. $\mathrm{Na}$ literatura apontada só um trabalho(6) tem grupo controle, portanto apenas este poderia usar o termo prevalência.

Neste estudo um paciente apresentou glaucoma de pressão normal. Mojon et al. ${ }^{(4)}$ encontraram em população suíça branca, 5 (7,2\%) casos de glaucoma sendo 3 primários de ângulo aberto e 2 de pressão normal.

No estudo de Bezerra ${ }^{(5)}$, que não faz referência à raça/cor, foram encontrados $4(4,4 \%)$ portadores de glaucoma mas os autores falam apenas em glaucoma de ângulo aberto não ficando claro se são glaucomas de pressão normal ou glaucomas primários de ângulo aberto. Deve ser ressaltado que neste estudo ${ }^{(5)}$ o diagnóstico de glaucoma foi feito por meio da análise de prontuários, sendo examinados apenas os 4 pacientes que apresentaram história positiva para glaucoma.

No estudo de Sergi et al. ${ }^{(6)}$ em pacientes brancos, e com grupo controle, os 3 casos $(5,9 \%)$ apresentaram glaucoma de pressão normal.

Apesar da patogênese do glaucoma de pressão normal ainda não estar bem definida, há evidências de que a hipoperfusão do nervo óptico causada por doenças isquêmicas crônicas e progressivas poderiam ser a cau$\mathrm{sa}^{(16)}$. A SAOS está associada com episódios de hipóxia prolongada, com excitação simpática aguda e prolonga- 
da compatível com aumento do tônus vascular. A SAOS aumenta a liberação de hormônios de ação vascular: catecolaminas, peptídeos natriuréticos atriais, angiotensina II, vasopressina e endotelina ${ }^{(13,14)}$. Assim a hipóxia prolongada característica da síndrome poderia causar dano direto ao nervo óptico. Outras possibilidades para explicar o dano ao nervo óptico seriam a alteração da autoregulação do fluxo sangüíneo na cabeça do nervo óptico ${ }^{(17)}$, secundária a apnéias prolongadas e repetidas ou secundária a hipertensão arterial e arterioesclerose $^{(18,19)}$ causadas pela própria SAOS ou ainda desequilíbrio entre óxido nítrico e endotelina ${ }^{(19)}$.

Chama atenção neste estudo o grande número de suspeitos de glaucoma, 9 (82\%) de 11 pacientes. Dos 9,5 tiveram o diagnóstico de suspeita de glaucoma por apresentarem à CTD picos pressóricos de até $32 \mathrm{mmHg}$ e grandes flutuações da PIO (PIO maior - PIO menor) de até $14 \mathrm{mmHg}$. Os picos pressóricos ocorreram sempre no horário das 6 horas da manhã quando o paciente estava deitado. Após levantar a PIO já sofria queda, mas manteve-se maior do que nos outros horários. A PIO elevada na posição supina poderia ser também outro fator contribuinte para o desenvolvimento do glaucoma nos pacientes com SAOS. Foi observada PIO aumentada em posição supina no horário das 6 horas em portadores de glaucoma tratados ou com hipertensão ocular ${ }^{(20)}$. Também em indivíduos normais observou-se PIO aumentada em posição supina, porém é maior nos pacientes com glaucoma de pressão normal( ${ }^{(21-23)} \mathrm{o}$ que pode reduzir o fluxo sanguíneo ocular nestes pacientes ${ }^{(24)}$.

Goldblum et al. ${ }^{(25)}$ avaliaram a PIO de 3 pacientes portadores de SAOS e glaucoma de pressão normal durante o sono em Laboratório do Sono. As avaliações da PIO foram feitas em 2 momentos: de respiração normal e no final de apnéias prolongadas sendo os valores semelhantes. Assim a PIO elevada em nossos pacientes é provavelmente postural e não por efeito da apnéia prolongada.

Não foi encontrado na literatura nenhum estudo do comportamento da PIO de 24 horas em pacientes portadores de SAOS. Apesar deste trabalho não ter grupo controle, os picos e as flutuações da PIO observados foram muito maiores do que o esperado na população normal. Deve ser ainda ressaltado que os cinco pacientes que apresentaram picos de $\mathrm{PIO}>21 \mathrm{mmHg}$ (números 1, 2, 7, 8 e 10) não apresentavam no momento do exame alterações de nervo óptico e/ou da camada de fibras nervosas.

Mesmo sendo glaucoma de pressão normal, o glaucoma mais frequentemente associado a SAOS, Sergi et al. ${ }^{(6)}$ encontraram PIO mais elevada nestes pacientes do que nos controles, o que sugere que também a PIO teria papel na fisiopatogenia do glaucoma associado à SAOS.

Fortalecem a relação glaucoma/SAOS não só a grande prevalência de glaucoma na SAOS, e a grande prevalência de SAOS (de $46 \%$ a $50 \%$ ) em portadores de glaucoma ${ }^{(7,9)}$, mas também a demonstração de Kremmer et al. ${ }^{(26)}$ de que a terapia com ventilação positiva nasal (CPAP) melhora o curso das duas doenças.

Este é um estudo observacional, assim como o são os estudos citados anteriormente e portanto não permite conclusões de causa e efeito da SAOS levando ao glaucoma, mas mostra associação da SAOS com glaucoma de ângulo aberto. Mostra ainda alto risco de desenvolvimento de glaucoma naqueles pacientes que apresentaram grandes picos pressóricos ${ }^{(27)}$ e grandes flutuações na avaliação da PIO de 24h, picos estes observados às $6 \mathrm{~h}$ com paciente deitado e portanto fora do horário da avaliação normal do consultório. As flutuações da PIO têm sido apontadas como importante fator de progressão do glaucoma ${ }^{(28)} \mathrm{e}$ o OHTS $^{(29)}$ mostrou que quanto maior a PIO e menor a espessura corneana maior é o risco de desenvolver glaucoma, o que sugere que os pacientes com PIO elevada terão maior probabilidade de desenvolver glaucoma.

Os resultados deste estudo em portadores de SAOS mostraram portanto alta freqência de glaucoma e de suspeitos de glaucoma. Apesar da amostra pequena e de não haver grupo controle, os dados sugerem que portadores de SAOS têm maior risco de desenvolver glaucoma do que indivíduos sem SAOS, e portanto deveriam ser submetidos à avaliação oftalmológica para pesquisa de glaucoma.

\section{Conclusão}

A SAOS pode ser um fator de risco importante para o desenvolvimento de glaucoma principalmente de pressão normal.

Pacientes com SAOS devem ser referidos para o oftalmologista e os oftalmologistas devem estar atentos para a associação de distúrbios do sono em pacientes com glaucoma de ângulo aberto.

\section{Abstract}

Purpose: To determine the frequency of glaucoma and evaluate the behavior of 24-hour intraocular pressure in patients with the obstructive sleep apnea syndrome (OSAS). Methods: Eleven consecutive patients with OSAS, diagnosed by polysonography, were avaliated in a cross sectional study. Demographic data were analyzed: age, sex, race/color, weight, height and associated diseases. The patients were submitted to complete ophthalmologic examination, including the visual field, as well as to 24hour intra-ocular pressure (IOP) evaluation by an applanation tonometer at $9 \mathrm{~h}, 12 \mathrm{~h}, 15 \mathrm{~h}, 18 \mathrm{~h}, 24 \mathrm{~h}$ and $6 \mathrm{~h}$ in the lying and sitting positions. The diagnostic criterion for 
glaucoma was alteration of the visual field (VF) compatible with glaucoma and one or more of the following alterations: cup-disc ratio $\geq 0.7$, hemorrhage, wedge-shaped defect, bayonet-shaped vessels, Hoyt's sign, asymmetry > than 0.2 between cup/disc ratio of the eyes. The angle should be opened without alterations. Results: $9(82 \%)$ of 11 patients showed glaucoma or were suspected to have glaucoma, $9 \%$ of which exhibited normal tension glaucoma and $73 \%$ were suspected to have glaucoma for presenting alterations in the optic nerve or ocular hypertension. The mean for the $I O P$ values of the 11 patients was observed to be the highest at $6 o^{\prime}$ 'clock, when they were lying down. Variations of IOP $\geq 5 \mathrm{mmHg}$ occurred in 7 (64\%) of the patients, and variations of up to $14 \mathrm{mmHg}$ and IOP peaks of up to 32 $\mathrm{mmHg}$ were observed. Conclusion: OSAS may be an important risk factor for the development of glaucoma, particularly that of normal tension glaucoma. Patients with OSAS must be referred to an ophthalmologist and those professionals must be attentive to the association of sleep disorders in patients with open-angle glaucoma.

Keywords: Sleep apnea, obstructive; Intraocular pressure; Glaucoma, open-angle; Diagnostic techniques, ophthalmological

\section{ReferêNCIAS}

1. Wilson MR, Martone JF. Epidemiology of chronic open-angle glaucoma. In: Ritch R, Bruce Shields M, Krupin T. The Glaucomas. 2nd ed. St Louis: Mosby; 1998. p. 753-68.

2. Weinreb RN, Khaw PT. Primary open-angle glaucoma. Lancet. 2004; 363(9422):1711-20.

3. Medeiros FA, Weinreb RN. Medical backgrounders: glaucoma Drugs Today (Barc). 2002; 38(8):563-70.

4. Mojon DS, Hess CW, Goldblum D, Fleischhauer J, Koerner F, Bassetti C, et al. High prevalence of glaucoma in patients with sleep apnea syndrome. Ophthalmology. 1999; 106(5):1009-12.

5. Bezerra ML. A prevalência do glaucoma nos pacientes com síndrome da apnéia/hipopnéia do sono do Instituto do Sono da Universidade Estácio de Sá. In: IX Congresso Brasileiro de Sono; 2003 Nov 5-8; Vitória. Anais. Vitória: Sociedade Brasileira de Sono; 2003. p. 45.

6. Sergi M, Salerno DE, Rizzi M, Blini M, Andreoli A, Messenio $\mathrm{D}$, et al. Prevalence of normal tension glaucoma in obstructive sleep apnea syndrome patients. J Glaucoma. 2007; 16(1):42-6.

7. Mojon DS, Hess CW, Goldblum D, Boehnke M, Koerner F, Gugger M, et al. Normal-tension glaucoma is associated with sleep apnea syndrome. Ophthalmologica. 2002; 216(3):180-4.

8. Mojon DS, Hess CW, Goldblum D, Bohnke M, Korner F, Mathis J. Primary open-angle glaucoma is associated with sleep apnea syndrome. Ophthalmologica. 2000; 214(2):115-8.

9. Marcus DM, Costarides AP, Gokhale P, Papastergiou G, Miller JJ, Johnson MH, Chaudhary BA. Sleep disorders: a risk factor for normal - tension glaucoma. J Glaucoma. 2001; 10(3):177-83.

10. Girkin CA, McGwin G Jr, McNeal SF, Owsley C. Is there an association between pre-existing sleep apnoea and the development of glaucoma? Br J Ophthalmol. 2006; 90(6): 679-81.

11. Geyer O, Cohen N, Segev E, Rath EZ, Melamud L, Peled R, et al. The prevalence of glaucoma in patients with sleep apnea syndrome: same as in the general population. Am J Ophthalmol. 2003; 136(6):1093-6.
12. Sleep-related breathing disorders in adults: recommendations for syndrome definition and measurement techniques in clinical research. The Report of an American Academy of Sleep Medicine Task Force. Sleep. 1999; 22(5):667-89.

13. Young T, Palta M, Dempsey J, Skatrud J, Weber S, Badr S. The occurrence of sleep- disordered breathing among middleaged adults. N Engl J Med. 1993; 328(17):1230-5.

14. Stradling JR, Crosby JH. Relation between systemic hypertension and sleep hypoxaemia or snoring: analysis in 748 men drawn from general practice. Br Med J. 1990; 300(6717):75-8

15. Strobel RJ, Rosen RC. Obesity and weight loss in obstructive sleep apnea: a critical review. Sleep. 1996; 19(2):104-15.

16. Hayreh SS, Zimmerman MB, Podhajsky P, Alward WL. Nocturnal arterial hypotension and its role in optic nerve head and ocular ischemic disorders. Am J Opthalmol. 1994; 117(5):603-24.

17. Hayreh SS. The 1994 Von Sallman Lecture. The optic nerve head circulation in health and disease. Exp Eye Res. 1995; 61(3):259-72.

18. Hayreh SS Acute ischemic disorders of the optic nerve. Pathogenesis, clinical manifestations, and management. Ophthalmol Clin North Am. 1996; 9:407-42.

19. Luscher TF. The endothelium and cardiovascular disease - a complex relation. N Engl J Med. 1994; 330(15):1081-3.

20. Rodrigues LD, Silva MR, Schellini SA, Jorge EN. Picos de pressão intra-ocular: comparação entre curva tensional diária, minicurva e medida da pressão intra-ocular às $6 \mathrm{~h}$. Arq Bras Oftalmol. 2004; 67(1):127-31.

21. Liu JH, Kripke DF, Twa MD, Hoffman RE, Mansberger SL, Rex KM, et al. Twenty-four-hour pattern of intraocular pressure in the aging population. Invest Ophthalmol Vis Sci. 1999; 40(12):2912-7.

22. Tsukahara S, Sasaki T. Postural change of IOP in normal persons and in patients with primary wide open-angle glaucoma and low-tension glaucoma. Br J Ophthalmol. 1984; 68(6):389-92.

23. Yamabayashi S, Aguilar RN, Hosoda M, Tsukahara S. Postural change of intraocular and blood pressures in ocular hypertension and low tension glaucoma. Br J Ophthalmol. 1991; 75(11):652-5

24. James CB, Smith SE. Pulsatile ocular blood flow in patients with low tension glaucoma. Br J Ophthalmol. 1991; 75(8):466-70.

25. Goldblum D, Mathis J, Böhnke M, Basset C, Hess CW, Gugger M, et al. [Nocturnal measurements of intraocular pressure in patients with normal-tension glaucoma and sleep apnea syndrome] Klin Monatsbl Augenheilkd. 2000; 216(5):246-9. German.

26. Kremmer S, Niederdraing N, Ayertey HD, Steuhl KP, Selbach JM. Obstructive sleep apnea syndrome, normal tension glaucoma, and nCPAP therapy - a short note. Sleep. 2003; 26(2):161-2.

27. Davanger M, Ringvold A, Blika S. The probability of having glaucoma at different IOP levels. Acta Ophthamol (Copenh). 1991; 69(5):565-8.

28. Asrani S, Zeimer R, Wilensky J, Gieser D, Vitale S, Lindenmuth $\mathrm{K}$. Large diurnal fluctuations in intraocular pressure are an independent risk factor in patients with glaucoma. J Glaucoma. 2000; 9(2):134-42.

29. Gordon MO, Beiser JA, Brandt JD, Heuer DK, Higginbotham EJ, Johnson CA, et al. The Ocular Hypertension Treatment Study: baseline factors that predict the onset of primary openangle glaucoma. Arch Ophthalmol. 2002; 120(6):714-20.

Endereço para correspondência:

Faculdade de Medicina de Botucatu - UNESP

Departamento de Oftalmologia, Otorrinolaringologia

e Cirurgia de Cabeça e Pescoço

Distrito de Rubião Júnior $\mathrm{s} / \mathrm{n}^{\circ}$

CEP 18618-970 - Botucatu - SP

Tel/fax: (14) 3811-6256 / 3811-6081

E-mail: rosabet@fmb.unesp.br 